\title{
Concomitant Medication Use Completion
} Status

National Cancer Institute

\section{Source}

National Cancer Institute. Concomitant Medication Use Completion Status. NCI

Thesaurus. Code C83234.

A term used to describe the state or condition of the completeness of the concomitant medication usage data. 